Itinéraires Itinéraires

Littérature, textes, cultures

2014-1 | 2015

Textualités numériques

\title{
L'écriture du fragmentaire quotidien entre mémoire discursive et mémoire métallique
}

Cristiane Dias

\section{OpenEdition}

Journals

Édition électronique

URL : http://journals.openedition.org/itineraires/2289

DOI : 10.4000/itineraires.2289

ISSN : 2427-920X

Éditeur

Pléiade

Référence électronique

Cristiane Dias, « L'écriture du fragmentaire quotidien entre mémoire discursive et mémoire métallique », Itinéraires [En ligne], 2014-1 | 2015, mis en ligne le 05 février 2015, consulté le 19 avril 2019. URL http://journals.openedition.org/itineraires/2289; DOI : 10.4000/itineraires.2289

Ce document a été généré automatiquement le 19 avril 2019

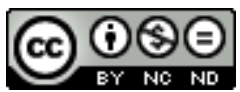

Itinéraires est mis à disposition selon les termes de la licence Creative Commons Attribution - Pas d'Utilisation Commerciale - Pas de Modification 4.0 International. 


\title{
L'écriture du fragmentaire quotidien entre mémoire discursive et mémoire métallique
}

\author{
Cristiane Dias
}

\author{
Amar o perdido \\ deixa confundido \\ este coração. \\ Nada pode o olvido \\ contra o sem sentido \\ apelo do Não. \\ As coisas tangiveis \\ tornam-se insensíveis \\ à palma da mão. \\ Mas as coisas findas, \\ muito mais que lindas, \\ essas ficarão.
}

(Carlos Drummond de Andrade, Memória ${ }^{1}$ )

\section{Espace d'écriture}

Dans Cybermigrances, Régine Robin attire notre attention sur « l'environnement de pensée qui supportait l'aventure hypertextuelle» des années 1980 (2004: 13). D’après elle, Barthes, Deleuze et Derrida ont travaillé dans la perspective de cet « environnement de pensée » et l'ont même constitué en explorant la fragmentation du texte (Barthes), les rhizomes (Deleuze), la lecture et l'interprétation (Derrida). Tous, à leur manière, ont poursuivi une «spatialisation du texte ", et même si, selon Robin, Derrida a poussé " plus loin les rapports de la pensée et du support d'inscription de celle-ci », ils ont été les précurseurs du mouvement de la littérature qui : 
a tendu vers la dislocation des formes traditionnelles, vers la discontinuité, la fragmentation, la ruine du sens, la dé-linéarité, la dé-séquentialité, la destruction de la totalité, voire de la totalisation. [Ce mouvement] a rencontré les pratiques formulaïques du roman populaire, celles du journalisme et du cinéma, de même que l'esthétique du montage et du collage. (Robin 2004 : 14) textuelles de représentation. Ainsi, pour Robin, avant l'ère actuelle de l'hypertexte submergé dans des logiciels, il y eut un temps premier, marqué par l'hypertexte de fiction, à une époque où les auteurs rêvaient d'une nouvelle expérimentation littéraire, libre des formes traditionnelles et des « formes textuelles de représentation» (2004: 18). Cependant, le désenchantement ne tarda pas. La manipulation de logiciels « de plus en plus lourds à maîtriser " prenait l'écrivain dans un « dispositif machinique qui est partie prenante de son écriture, des mécanismes narratifs qu'il utilise, et [il] devient un cyborg en symbiose avec la machine ». Dans cette conjoncture, le texte semble se perdre dans les pratiques de la cyberculture, dans la «nouvelle culture, [des] pratiques du chat, de l'immédiateté, de l'e-mail et de la communication à l'économie de langage, quand ce ne sont pas les messages brefs, les textes formés à l'imaginaire des jeux vidéo, à l'interactivité » (2004: 19).

3 Autrement dit, les pionniers de l'hypertexte ont compris que la spécificité des nouveaux médias exigeait aussi une autre culture, puisque l'écriture sur le Web, l'écriture numérique est régulée par d'autres imaginaires, d'autres relations entre l'auteur et le lecteur, entre le texte et le moyen de production. Dans la mesure où le support change, les conditions de production littéraire deviennent autres. De fait, toute variation de la forme matérielle ${ }^{2}$ se reflète nécessairement sur les sens, le rapport à la mémoire et la manière dont la narrativité est construite.

4 Avant même l'âge de l'hypertexte et du changement culturel affectant l'écriture, quelques écrivains des années 1920 s'étaient déjà interrogés sur les transformations de la forme narrative engendrées par la culture de masse et ses médias. Ces transformations dans la forme matérielle bouleversent également la notion de mémoire, une fois qu'elles modifient l'historicité du sens.

5 Il suffit de lire le chapitre de La Mémoire saturée de Régine Robin (2003) où l'auteure aborde la mémoire et les médias en référence à la pensée de Walter Benjamin et de Siegfried Kracauer sur les médias de masse de leur temps pour se demander, un peu comme Derrida l'avait fait quant à la forme que la théorie freudienne aurait prise à l'époque de l'e-mail (2001), ce que les mémorialistes, observateurs et chroniqueurs de la culture de masse, comme les appelle Robin (2004), diraient de l'âge du numérique et de la constitution de la mémoire dans ce contexte.

6 Cette question, et plus particulièrement ses aspects concernant la mémoire et l'écriture de soi, nous invite à développer, dans cet article, une réflexion sur l'écriture numérique sur le réseau social Facebook et sur le changement de construction de la forme narrative.

7 Ce dernier sera considéré à partir de ce qu'Orlandi (2004a) ${ }^{3}$ a appelé la «narrativité de l'urbain ", qui, selon elle, est le produit d'un désordre de l'espace bureaucratique de l'urbain et comprend des scènes qui ne sont pas rapportées par un narrateur, puisque la ville n'en a point: le récit fait partie de la scène. La "narrativité de l'urbain » est constituée des mots de la ville dans sa matérialité dispersée. 
8 À partir de cette notion, nous proposons une approche de la " narrativité du numérique » sur Facebook, c'est-à-dire de ce que les sujets y révèlent de leur vie, dans des textes, des parcours ou des partages - ce que nous appelons l'« écriture du fragmentaire ».

\section{La mémoire métallique}

Dans son ouvrage Interprétation (1996), Orlandi propose pour la première fois la notion de «mémoire métallique» afin d'appréhender théoriquement le fonctionnement des technologies du langage basées sur l'usage de l'ordinateur et d'Internet.

Dans une interview de 2004 (2004b), elle affirme que la mémoire métallique est celle produite par les automates, les machines, bref, un simulacre de mémoire. Au contraire du fonctionnement vertical de la mémoire discursive, où il y a filiation du sujet à un réseau de mémoire qu'il actualise dans une formulation pour signifier, le fonctionnement horizontal de la mémoire métallique ne produit que la somme de ses énoncés, sans filiation, en une actualisation automatique.

11 Si un énoncé, une image ou une vidéo a un effet viral, c'est « un dire toujours réactualisé [...] ne faisant qu'effet de mémoire ». La mémoire métallique fonctionne "comme si elle était mémoire" (Orlandi 2004b: 26-27). C'est une illusion de mémoire qui ne fait qu'accumuler le sens sans l'historiciser. L'aspect le plus courant de la viralité est la circulation. Or, quand un dire circule dans la matérialité numérique, il signifie du fait même qu'il circule. Voilà pourquoi la mémoire métallique implique une évidence du sens et une transparence du sujet, car le processus de signification est contrôlé par l'existence technique de l'énoncé et de toutes les possibilités de liens que cette dernière peut engendrer, comme les posts les plus partagés ou les trending topics ${ }^{4}$, par exemple.

Comprendre la mémoire métallique et ses enjeux est donc fondamental pour appréhender la narrativité du numérique à partir de la constitution de ce que nous appelons le fragmentaire d'une vie.

13 Il est donc permis de nous demander quels effets de sens les réseaux sociaux produisent dans la constitution de ces fragmentaires d'une vie ordinaire et comment le sens de la vie écrite se modifie en fonction d'une mémoire (de la machine) qui ne cesse d'archiver. Cette « image archivale ", Robin la comprend comme un «aide-mémoire » produisant une vie artificielle (2003: 387), dans la mesure où les images archivées se «détachent de leur agencement originel, de leur contexte spatial » (2003 : 389). Elle s'oppose, toujours selon Robin, à celle de l'expérience définie par Walter Benjamin et à la mémoire involontaire de Proust, laquelle est un «tissage de souvenirs" n'ayant rien à voir avec leur enregistrement: c'est un jeu de souvenirs et d'oublis qui nous donne la possibilité de remplir les marges, de retoucher la vie et les événements. La mémoire archivale, par contre, fige le souvenir dans la « sphère de l'événement vécu » $(2003: 389)$.

Dans cette perspective, nous pouvons affirmer que la circulation des événements sur les réseaux sociaux produit un effet de saturation du sens, comme dans un jeu où ce qui est mis à jour est un enregistrement, une archive sans mémoire, parce que construite sans marges, dans un but de réplication.

15 Cependant, la reproduction technique d'une vie racontée l'insère dans l'ordre de la transparence du sens et produit un effet de complétude, ce qui constitue en même temps un effet pervers du fonctionnement de la mémoire métallique, lequel est dû au rapport établi par le sujet avec une mémoire qui accumule et où tout se tient. D'où l'importance 
du concept de mémoire métallique, c'est-à-dire de mémoire produite par les médias, par les nouvelles technologies du langage. Une mémoire de l'information impliquant « quantité et non-historicité » (Orlandi $2006: 5$ ).

16 Si la mémoire discursive ${ }^{5}$ se constitue par l'oubli et la mémoire de l'archive par le nonoubli, puisqu'elle est contrôlée par une normalisation du processus de signification, comme nous l'enseigne Orlandi (2006), la mémoire métallique se constitue par l'excès, par la quantité. Elle n'oublie pas, ni même n'institutionnalise ni ne normalise pour ne pas oublier, mais additionne, accumule, raison pour laquelle nous la comprenons comme une mémoire numérique.

Dans cette perspective, les différentes formes de mémoire soulèvent des questions fondamentales pour penser une écriture du numérique, du fait que, pour la mémoire métallique, l'espace de circulation des sens est un espace virtuel où tout se tient, où rien ne manque, et où l'archive devient éternelle.

18 Cette notion de mémoire métallique, distincte de la mémoire discursive et de la mémoire d'archive, nous permet donc de comprendre comment l'écriture du quotidien sur les réseaux sociaux produit du sens à partir de différentes formes de mémoire et d'interprétation. Orlandi considère ainsi que la mémoire possède différentes matérialités (2006).

N'oublions pas que les conditions de production de l'écriture contemporaine relèvent en grande partie de l'ordinateur et d'Internet; que la mémoire métallique est liée à la quantité, à l'excès et aux médias ; que la mondialisation est associée à l'effacement des frontières et à la déterritorialisation du texte qui tisse la vie quotidienne, au présent ou au passé, aussi bien que l'histoire et le rapport à la mémoire des événements ordinaires d'une vie. Nous pouvons donc parler d'une écriture du fragmentaire empruntant des éléments de la vie commune, constituée de ce que les sujets racontent de leur vie sur Facebook, soit d'une écriture du présent, de l'instant.

20 Ce qu'Orlandi nous dit des «formes du discours urbain » nous permet d'affirmer que le « fragmentaire » est compris comme « effet de la volonté de totalité » (2004: 29). En effet, selon elle, la matérialité de la ville est ce qui désorganise le lieu totalisateur de l'organisation urbaine dans la mesure où elle oblige le sujet au parcours. Ainsi, c'est le parcours de ce que le sujet dit de lui-même dans ses « fragmentaires » - textualisations de soi - qui fait que Facebook organise l'incomplétude et la dispersion du sujet en une linéarisation temporelle du dire : liens, partages, posts, etc., soit une « ligne du temps ».

21 Voici un exemple de cette écriture du fragmentaire : 
Fig. 1. Exemple d'écriture fragmentaire sur Facebook

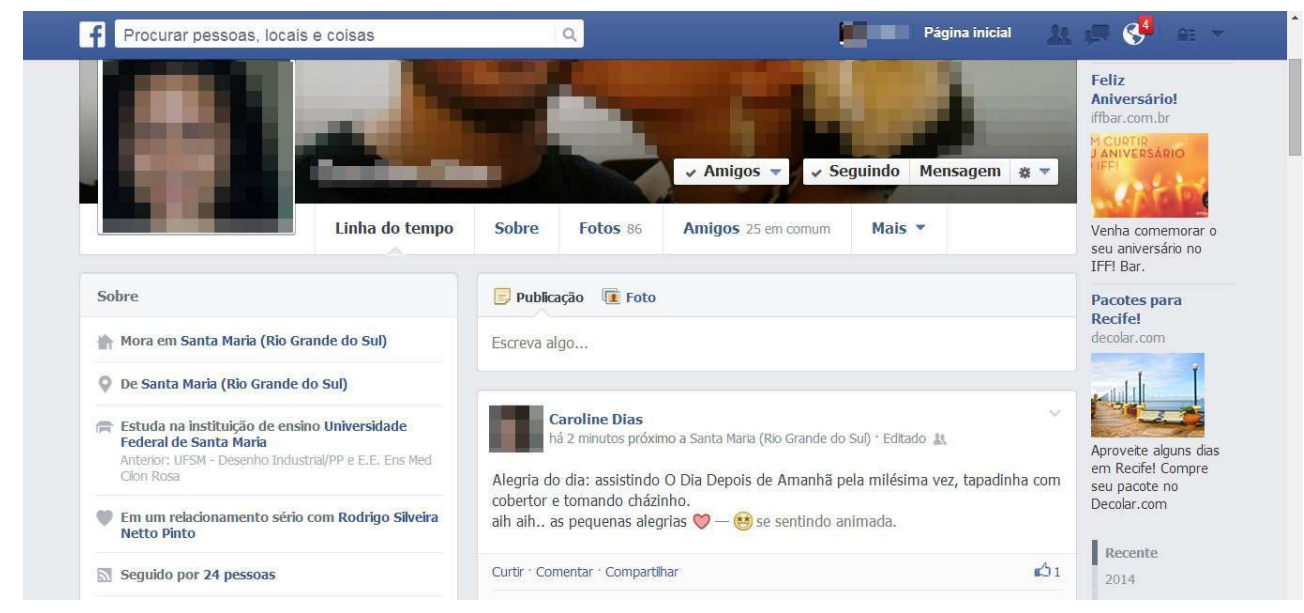

Le fragmentaire ci-dessus nous montre une textualisation de soi commençant par l'annonce d'une synthèse de la totalité d'un jour. Le sujet écrit : «Bonheur du jour ", ce qui pour lui consiste à regarder un film sous une couverture en buvant du thé, et termine le texte par une autre synthèse : «ah... les petits bonheurs ", par rapport à une totalité plus grande qu'est la vie. «Bonheur du jour » et « les petits bonheurs de la vie » sont les fragmentaires signifiés par une séquentialité événementielle : regarder un film, être sous la couverture et boire du thé. Dans une écriture du fragmentaire toute une vie est synthétisée en cette linéarisation temporelle du dire, effet de la volonté de totalité.

Tous les éléments de l'exemple ci-dessus font partie d'une narrativité : les photos, les informations personnelles à gauche, les publications. À droite de l'image, il existe une possibilité de navigation hypertextuelle du post le plus récent - 2014 - à la «naissance » du profil sur Facebook. On peut se rendre à n'importe quel point de la ligne du temps du sujet, en choisissant une année ou un mois. Ce sont ces nœuds du temps qui organisent la narrative dans la dispersion du réseau, chacun marquant l'unité du sujet. Le mariage, la fin des études, l'obtention d'un emploi sont autant de points fixes de la narrative signés par un événement-nœud. 


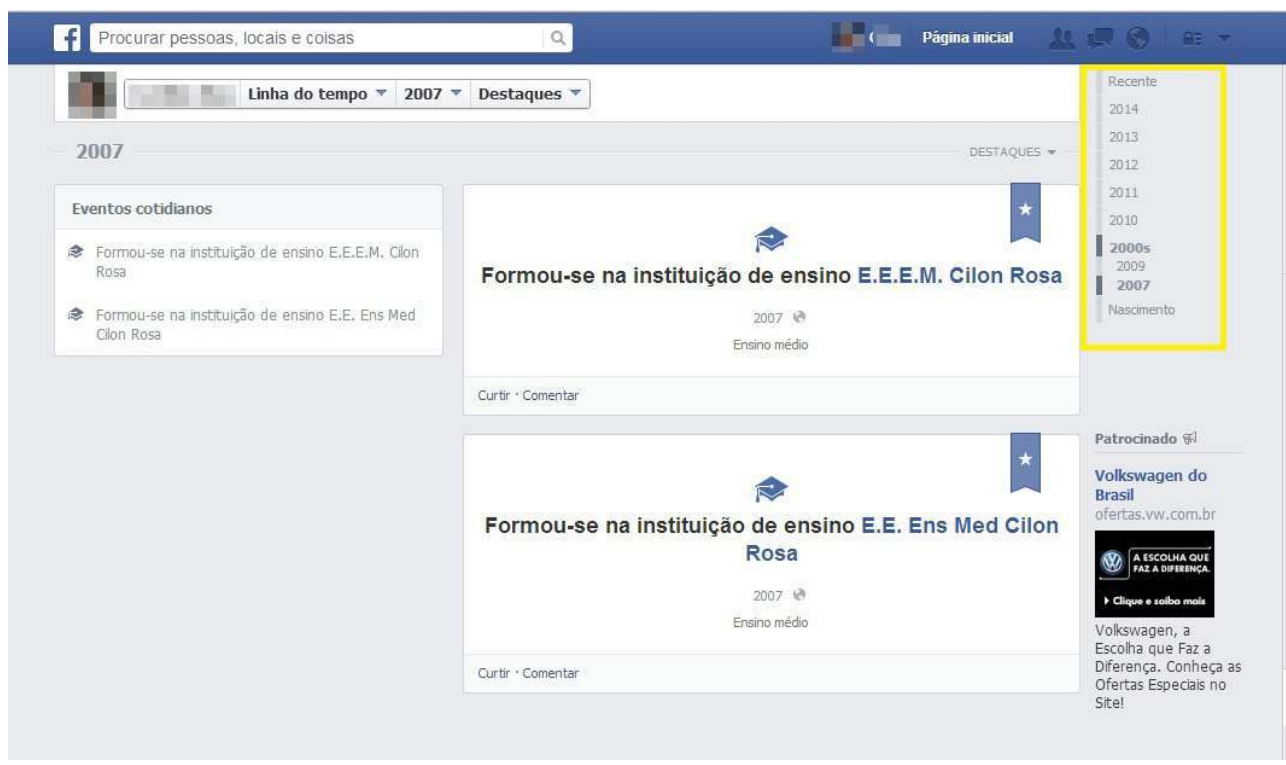

Le parcours du sujet via cette écriture du fragmentaire constitue ce que nous appelons la narrativité du numérique, selon laquelle les sens ne peuvent être compris que dans leurs fulgurations sur la ligne du temps.

\section{La mémoire du futur}

La vitesse est l'une des caractéristiques de l'écriture du fragmentaire, de cette écriture des rapports qui construit la narrativité en une "matérialité dispersée » (Orlandi 2004a). Par conséquent, comprendre le temps sous l'aspect de sa matérialité nous permet de comprendre le fonctionnement des différentes formes de mémoire.

Dans cette perspective, penser les effets de ce fonctionnement de la forme d'écriture sur la constitution du sujet implique de penser les modifications des conceptions du temps mais aussi de l'espace qui sont en jeu dans le numérique.

Le temps - ou le contrôle du temps - est une forme de pouvoir, et il est amplement exploité dans la société numérique. L'accélération du temps, marquée par la vitesse des réseaux, conduit le sujet vers le futur car il y projette sa mémoire, son corps, sa place politique, et même sa vie, en effaçant le présent. En ce sens, sa vie au présent a très peu de valeur si elle peut être archivée pour le futur, pour les prochaines générations, pour la vie artificielle. Ainsi, le stockage de la mémoire est la mémoire du futur, ce qui donne au sujet l'illusion de fuir le réel: la mort, la disparition, l'oubli. Il s'agit de la mémoire archivée pour le moment où le sujet ne pourra plus se rappeler, ne sera plus présent, mais ressuscitera d'entre les morts.

Du point de vue technique il y a deux types de mémoire du futur: celle de l'expérimentation technique programmée pour le stockage, but du sujet, et celle de l'expérimentation spontanée, où le stockage est le but de la machine. Par exemple, dans les expériences d'enregistrement programmé réalisées par des équipements et des logiciels, telles qu'elles sont décrites par les scientifiques américains Gordon Bell et Jim Gemmel dans leur essai Total Recall (2009), la mémoire du futur est programmée, puisque 
des équipements spécifiques sont préparés pour consigner la vie des scientifiques, à la manière d'un big brother privé et autoréflexif. Par contre, dans l'enregistrement de la mémoire des réseaux sociaux, comme Facebook, la mémoire du futur est spontanée, c'està-dire que son stockage, même s'il a lieu, n'est pas le but du sujet. Il faut ajouter que l'usage de dispositifs mobiles, comme les téléphones portables, les caméras numériques, ou de logiciels comme Instagram, fait partie de cette culture de la mémoire du futur.

Cette culture de l'usage d'appareils mobiles a des effets sur le mode de vie contemporain puisqu'elle conduit à la constitution d'un sujet toujours en manque, n'envisageant plus le paysage qu'à travers l'écran de sa caméra numérique ou de son ordinateur. Les équipements et les logiciels font partie d'une forme de mobilité permise par la numérisation du monde et produisent un espace-mémoire ou un espace comme espèce de mémoire.

Pour mieux comprendre cet espace-mémoire, nous évoquerons les deux métaphores importantes souvent employées par les théoriciens du cyberespace : celle du labyrinthe et celle de la navigation. Elles visent à expliquer un certain fonctionnement du cyberespace, l'une par sa non-linéarité, l'autre par sa fluidité, et sont construites sur une mémoire mythologique ainsi que sur une mémoire discursive de l'espace politique et économique de la navigation, respectivement. Ces deux modes différents de déplacement, mais aussi de lecture et d'écriture, ont bouleversé l'écriture littéraire, comme le prouvent les récits labyrinthiques permettant au lecteur de choisir son parcours de lecture, soit par des liens, soit par des réponses à des questions posées par l'auteur au cours de la construction narrative. Comme nous l'avons évoqué plus haut, ces possibilités ont été expérimentées dès les débuts de l'hypertexte, quand certains écrivains se sont aventurés dans une écriture non linéaire.

31 Cependant, quels sont les parcours et les déplacements impliqués par la forme de narrativité de l'âge du numérique ? Comment nous mouvons-nous dans le tissu scriptural du numérique, constitué de réseaux dont la topologie logique peut se reconfigurer à tout instant, grâce à des équipements comme des routeurs portables par exemple? Quels sont les itinéraires des sujets sur ces réseaux sociaux? Quel rapport à l'espace les sujets éprouvent-ils?

Dans cette mise en scène de la mémoire du futur, il est intéressant d'observer un fonctionnement du parcours que nous qualifierons de narratif puisque les informations fournies par les sujets (photos, SMS, vidéos, etc.) et stockées par la machine - produisant la mémoire métallique - peuvent être actualisées à tout moment sur la ligne du temps, soit par un commentaire soit en cliquant sur "J'aime». Cette actualisation narre une histoire renouvelée dans le temps. Ainsi, le caractère ouvert de ce que les sujets racontent est l'un des aspects de la narrative du fragmentaire. 
Fig. 3. Narrativité du numérique

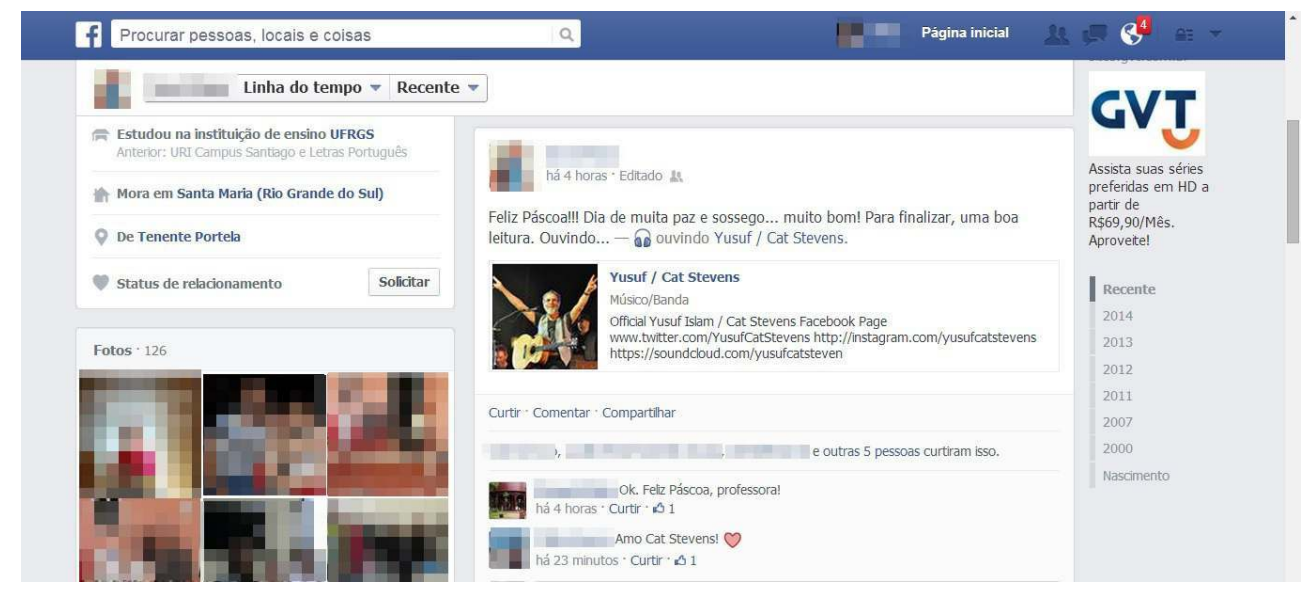

Dans ce cas, le narratif se configure par : 1) l'actualisation cliquante ;2) le stockage 3); le rapport du sujet à une mémoire métallique ; 4) l'organisation algorithmique du temps ; 5) les activités (commentaires, j'aime) concernant une publication (plus un post est partagé ou aimé, plus il atteint son objectif de réplication dans la mémoire métallique).

Si nous prenons en compte le changement de forme de la construction narrative précédemment mentionné, nous pouvons parler d'une narrativité du numérique composée à l'aide de logiciels, d'équipements et de périphériques, comme les portables (qui peuvent fonctionner comme des routeurs et reconfigurer la topographie logique des réseaux) et les appareils photo numériques. L'usage constant de ces dispositifs automatise, d'une certaine façon, la narrativité du quotidien, la poétique du monde en produisant une mémoire métallique.

Ces appareils électroniques et numériques font partie de ce qu'Orlandi (2004a) appelle la « narrativité urbaine », mais aussi, pourrions-nous dire, de la narrativité e-urbaine, c'està-dire de l'espace urbain constitué par une discursivité du numérique. Cela implique une forme de mobilité déterminée par le numérique, qui se manifeste par les caméras de surveillance, les produits porteurs de codes QR, l'accessibilité portable d'Internet, le réseau Instagram qui enregistre le « temps réel » et le diffuse sur les réseaux sociaux, etc. Tout ce mouvement technique de l'urbain que nous appelons la mobilité du numérique, soutenue par la mémoire métallique, «c'est le temps du capitalisme, de l'immédiateté, effet idéologique qui, en même temps, construit un sujet libre et responsable et le signifie par l'irresponsabilité » (Orlandi 2012:17).

36 Ces possibilités technologiques de sujets simulés, d'avatars, de semblants de passé ou de futur exigent une analyse des effets d'une conception du sujet simulé constitué par rapport à une mémoire métallique, soit un sujet qui se contredit dans le temps de l'histoire, de la vie sociale et politique et se signifie dans le temps des algorithmes.

Tout changement de l'algorithme de Facebook, par exemple, modifie la forme de narrativité et fait apparaître une facette technique de la vie racontée où les moyens numériques transforment les liens sociaux et symboliques, requalifient les corps et les affectent. Dans les rapports humains établis sur les réseaux sociaux, le corps est simulé, numérique. Par conséquent, l'un des effets de la création d'un corps numérique, sans mémoire historique ni oubli, est la totalisation de la mémoire. 

et désigne "tout dispositif qui stocke et retrouve à loisir de l'information" " (2007: 401). Or, cette mémoire informatique, qui s'impose par la discursivité de la technologie, affecte le sujet dans sa pratique et ses rapports quotidiens, puisque le contact avec l'ordinateur implique la mise en relation du sujet avec des milliards de données par seconde. Ce langage de l'informatique, déterminé par une série d'opérations et d'instructions techniques (le calcul, l'interprétation et le traitement des instructions), fait partie de la vie quotidienne du sujet à travers un processus très familier dans notre société, la vitesse. L'accélération du temps est l'une des grandes questions actuelles concernant la façon dont on produit, formule et fait circuler les connaissances dans les médias, dans les institutions d'enseignement ou dans notre quotidien. Mais la vitesse concerne aussi la façon dont on établit des liens sociaux avec l'autre. l'informatique matérialisés dans les processeurs, il n'en est pas moins intéressant d'aborder la question de l'espace, c'est-à-dire de la mémoire morte et de la mémoire vive. $\mathrm{Si}$, d'un côté, Dubey (2001) parle d'une instrumentalisation des affects et des liens sociaux, de l'autre, Robin (2003) parle d'une instrumentalisation de la mémoire. Dans ces deux cas, la numérisation de nos rapports au temps et à l'espace est la base de l'instrumentalisation. Il convient donc de se demander si la mémoire est une question de temps ou d'espace. D'un point de vue analytique et discursif, si l'on considère l'effet métaphorique et l'historicité du sens de la mémoire métallique, on note une dérive du sens de la mémoire du temps, qui se constitue par l'oubli, vers la mémoire de l'espace, qui se constitue par le stockage. Plutôt que de l'abolition du temps et de l'espace, il s'agit de la prédominance de l'espace sur le temps, pour ce qui est de la notion de mémoire. Dans cette perspective, Robin attire notre attention sur les métaphores utilisées pour désigner les capacités de stockage : « Elles ont toutes trait à la mémoire » (2003:407).

En réalité, il est question de temps et d'espace, car dans ce travail d'extension de la mémoire morte de l'ordinateur, c'est le temps que l'on veut garder, stocker, arrêter et redémarrer (par l'actualisation de la mémoire vive) ou accélérer. Arrêter le temps, le contrôler, les hommes en rêvent depuis toujours. Avec l'informatique, cependant, il est possible d'arrêter le temps de la machine. D'après Herrenschmidt : « Ce temps n'est pas le temps des hommes, continu et durable, c'est une pure scansion dont la cadence est déterminée par la puissance du processeur " (2007 : 402). La métaphore de la navigation et du labyrinthe décrivant le monde numérique produit en effet une équivoque : elle exprime la fluidité et la non-linéarité du temps, alors que le numérique instaure au contraire une durabilité rigide, non fluide.

41 Mais une question centrale concerne les rapports sociaux, affectifs : cette nature de la mémoire les modifie-t-elle? Et de quelle manière? Nous avons vu plus haut que, si la mémoire discursive est constituée par l'oubli et la mémoire d'archive par l'absence d'oubli, la mémoire métallique, pour sa part, se compose d'excès et de quantité (Orlandi 2006). Prise comme espace de stockage, elle est alors réduite à une « construction technique » qui ne s'érige pas dans l'historicité. Elle n'est qu'un traitement de données, pas un processus historique. Voilà peut-être l'une des conséquences les plus importantes de la conception de la mémoire comme espace (de stockage) et non pas comme temps (d'oubli). En effet, d'après Orlandi :

La modalité de circulation du sens dans le discours électronique nous fait penser que, par sa spécificité, elle produit des conséquences sur la fonction-auteur et sur 
l'effet-lecteur. Ces conséquences sont directement liées à la caractéristique de la mémoire. Et, sûrement, à la matérialité signifiante de ses moyens ${ }^{6}$. (2006:5)

Ce sont ces conséquences sur la fonction-auteur et sur l'effet-lecteur liées à la mémoire qui nous intéressent pour comprendre le fonctionnement discursif d'un fragmentaire de vie partagé sur Facebook.

\section{L'écriture du fragmentaire}

$\mathrm{Au} \mathrm{XXI}^{\mathrm{e}}$ siècle, la navigation cliquante sur le Web et, plus particulièrement, une écriture numérique fondée sur le collage d'images, de sons, de mots, de vidéos, composant le fragmentaire d'une vie, tissent des réseaux de rapports numériques marquant la poétique du monde et construisent une narrativité.

Ce tissu de relations est soutenu par un partage d'informations, de souvenirs, de photos, de vidéos, de SMS, etc. Sur Facebook, une infinité de fragmentaires circulent, qui sont traversés par une mémoire métallique produisant ce que nous avons appelé un enregistrement spontané de la mémoire, lequel peut produire des effets de sens déjà prévus par Robin, c'est-à-dire "un enregistrement fidèle des données [qui] viendrait bloquer le travail de cette mémoire vivante, lui substituant une mémoire artificielle, incapable de suppléer à ces manques, une mémoire pleine » (2003:394).

Examinons le post suivant sur Facebook, en considérant le fonctionnement de la mémoire prise comme espace (stockage), dans sa contradiction avec la mémoire prise comme temps (oubli).

Fig. 4. La « mémoire-circulation »
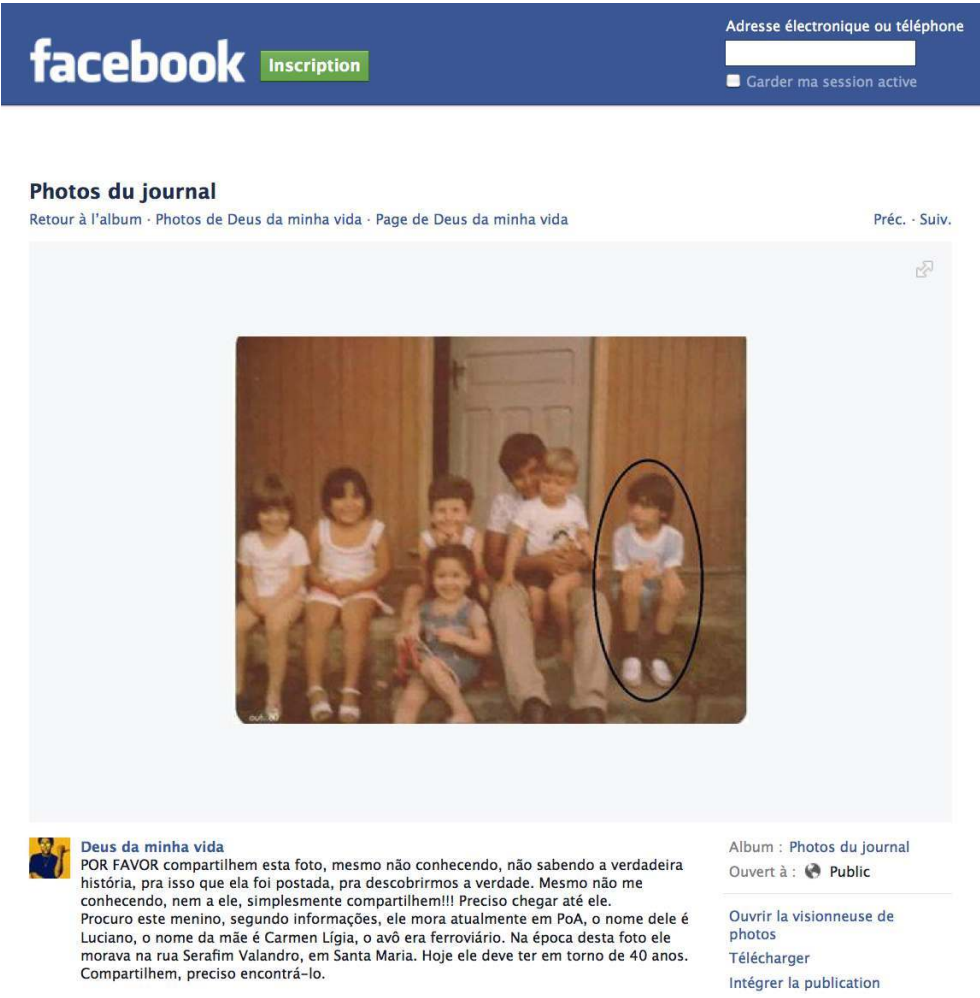

même s'ils ne connaissent pas la véritable histoire, pour découvrir la vérité sur l'enfant cerclé, à droite. Le statut dit à peu près :

Je recherche ce petit garçon, selon mes informations il habite actuellement à Porto Alegre. Il s'appelle Luciano, sa mère s'appelle Carmen Lígia, son grand-père était cheminot. Quand cette photo a été prise, il habitait rue Serafim Valandro, à Santa Maria. Aujourd'hui, il a à peu près 40 ans. Partagez, j'ai besoin de le retrouver.

Le narrateur nous révèle un certain parcours du sujet de la narrative, ce qui construit en même temps un enchaînement narratif du point de vue syntagmatique.

La photographie montre des enfants assis devant une maison dans un passé non précisé, mais marqué dans le texte par la formulation: «quand cette photo a été prise ». Nous connaissons aussi la localisation géographique de la maison de la photo grâce à des marqueurs de temps et d'espace: Santa Maria, une ville au sud du Brésil, et plus précisément la rue Serafim Valandro. En ce qui concerne le temps présent du partage, nous avons aussi des déictiques : «il habite actuellement à Porto Alegre ». Nous pouvons encore identifier un autre marqueur de temps, "aujourd'hui », qui peut être compris comme indéfini aussi bien par rapport au temps de la photo que par rapport au temps de son partage, lequel marque justement le présentisme : l'impossibilité de retrouver ce petit garçon.

Ces éléments de référence au temps et à l'espace du texte nous permettent de souligner les effets de la mondialisation, de l'effacement des frontières et de la déterritorialisation du texte que nous avons évoqués au début de cet article. Si l'on considère le réseau social sur lequel la photo a été partagée, avec ses conditions de production et sa topographie en réseau de nœuds à l'infini, on se rend compte qu'il est difficile de savoir qui a partagé la photo et quand elle a été partagée. Tout ce que nous savons, c'est qu'elle circule sur Facebook, et c'est le fait même de cette circulation qui produit son sens et sa temporalité.

Cela signifie que les marqueurs de temps auxquels nous sommes habitués, à savoir, le présent, le passé, le futur, en rapport historique et en référence à un événement faisant sens dans l'histoire à partir de certaines filiations du sujet, ne sont pas, dans cette modalité de production du sens traversée par la mémoire métallique, des éléments constitutifs : dans la circulation comme spatialisation instantanée de la mémoire, le temps est toujours présent, c'est le "présentisme » dont parle Orlandi (2012), l'image équivoque du temps réel.

51 Dans ce dispositif, les marqueurs du temps (quand cette photo a été prise, aujourd'hui, actuellement) de la mémoire métallique fonctionnent par rapport à la spatialisation du texte sur l'écran et non par rapport à l'historicisation d'un événement.

52 La photo, partagée dans le flux de nouvelles de Facebook, attire l'attention sur l'instant, particulièrement du fait de la sélection d'un des enfants. Tous semblent poser pour la photo, sauf celui que l'on recherche. Lui ne sourit pas et regarde les autres comme s'il ne faisait pas partie de la scène. Pourquoi le recherche-t-on? Qui le recherche? Autant de questions auxquelles il est difficile de répondre, puisque la topographie de Facebook se constitue par le partage ou la duplication des images, textes, informations, etc.

Ce que cette photo nous présente, c'est bien la chose répliquée. Mais quel est son lien avec le temps établi par le geste de partage de cette photo sur Facebook? Quel rapport avec la mémoire ce lien établit-il ? Et qui est l'enfant recherché sur cette photo? Quelle est sa "véritable " histoire? Aucune réponse. "La photographie devient fantôme ", déclare 
Kracauer, " elle représente ce qui est absolument passé, mais ce déchet a été un présent " (2008: 52). Et c'est à partir de ce déchet que le sens échappe. "Polysémie et silence ${ }^{7}$ ", « sens en fugue », comme le dit Orlandi, c'est-à-dire « explosion qui déstabilise et produit un mouvement désordonné » (25).

Tous les partages de cette même photo font exploser le sens en fragmentaires d'une vie ordinaire du passé qui s'actualise dans notre flux sur Facebook, comme le flash d'un appareil photo qui disparaît vite au milieu de dizaines d'autres éclairs, pour composer une narrativité du numérique sur notre écran. C'est précisément cette fugue du sens qui met en marche la mémoire discursive ou la mémoire de l'événement, différentes d'une mémoire de l'espace. Cette mémoire qui se détache de la mémoire métallique de la chose répliquée, en tension, après que l'usager de Facebook a partagé une photo dont il ne sait rien, c'est l'irréalisable.

\section{BIBLIOGRAPHIE}

Bel, Gordon et Gemmel, Jim, 2009, Total Recall: How the E-Memory Revolution Will Change Everything, Boston, Dutton.

Derrida, Jacques, 2001, Mal de arquivo: uma impressão freudiana, trad. Claudia de Moraes Rego, Rio de Janeiro, Relume Dumará.

Dubey, Gérard, 2001, Le Lien social à l'ère du virtuel, Paris, PUF.

Herrenschmidt, Clarisse, 2007, Les Trois Écritures : langue, nombre, code, Paris, Gallimard.

Kracauer, Siegfried, 2008, Le Voyage et la Danse. Figures de ville et vues de films, Québec, Presses de l'Université Laval, coll. «Pensée allemande et européenne ».

Orlandi, Eni Puccinelli, 1996, Interpretação: autoria, leitura e efeitos do trabalho simbólico, Rio de Janeiro, Vozes.

-, 2001, Discurso e texto, Campinas, Pontes.

-, 2004a, Cidade dos sentidos, Campinas, Pontes.

-, 2004b, « História das ideias x história de vida: entrevista com Eni Orlandi », Fragmentum, n 7 , [En ligne], DOI : 10.5902/21792194.

-, 2006, « Análise de discurso: conversa com Eni Orlandi », Teias, vol. 7, n $13-14$, interview à Raquel Goulart Barreto, [En ligne], http://www.periodicos.proped.pro.br/index.php/ revistateias/article/view/210.

-, 2012, "Sentidos em fuga: efeitos da polissemia e do silêncio », dans Guilherme Carrozza et al. (dir.), Sujeito, sociedade, sentidos, Campinas, RG Editora, p. 1-15.

Pêcheux, Michel, 1999, « Papel da memória », dans Pierre Achard et al., Papel da memória, trad. José Horta Nunes, Campinas, Pontes, p. 49-57.

Robin, Régine, La Mémoire saturée, Québec, Stock, 2003.

-, 2004, Cybermigrance : traversées fugitives, Québec, VLB éditeur, coll. « Le soi et l'autre ». 


\section{NOTES}

1. «Aimer ce que l'on a perdu / Laisse ce cœur-là confus. / Ce que l'on a oublié / Ne peut rien contre l'insensé / Appel du non. / Les choses tangibles / Deviennent insensibles / Sous la paume de la main / Mais les choses révolues / Bien plus que belles, / Elles resteront. »

2. Orlandi (2001) a redéfini dans une perspective discursive la notion de «forme matérielle » en la distinguant de la notion de forme matérielle de Hjelmslev, lequel travaille sur une distinction forme de l'expression/forme du contenu, sens. Dans ses travaux, Orlandi ne fait pas de distinction entre forme et contenu. «Les sens sont tels qu'ils se constituent, se formulent et circulent »(12), affirme-t-elle. Dans cette perspective, la forme matérielle du langage est linguistique/historique. C'est la forme en tant que processus.

3. Toutes les citations d'Orlandi sont traduites du brésilien par mes soins.

4. Les trending topics sont des mots ou des hastags classés dans une liste en temps réel des tweets les plus utilisés et/ou disseminés sur le réseau social Twitter.

5. D'après Pêcheux (1999), la mémoire discursive est ce qui, dans un texte, apparaît comme un événement à lire, qui vient rétablir les implicites dont la lecture a besoin (c'est-à-dire, plus techniquement, les préconstruits, éléments rapportés, discours-transverses, etc.).

6. Eni Orlandi propose, par la notion de matérialité signifiante, de montrer que les formes ne sont pas indifférentes à la matière signifiante. Par exemple, la peinture, la photographie, l'écriture, etc., constituent différentes matérialités signifiantes qui affectent l'interprétation en lui donnant une forme.

7. Nous faisons ici allusion à la réflexion proposée par Orlandi : «Sens en fugue : effets de la polysémie et du silence » (2012), où elle considère la fugue dans son sens musical, plutôt que dans son sens commun, soit comme « forme complexe de composition polyphonique (polysémique?) fondée sur un thème présenté en plusieurs modalités » (11).

\section{RÉSUMÉS}

$\mathrm{Au} \mathrm{xxI}^{\mathrm{e}}$ siècle, la navigation cliquante sur le Web et, plus particulièrement, une écriture numérique fondée sur le collage d'images, de sons, de mots, de vidéos composant le fragmentaire d'une vie tissent des réseaux de rapports numériques qui marquent la poétique du monde. Or, ces réseaux nous intéressent dans la mesure où la matérialité de ce tissu écrit est le quotidien, la vie quotidienne, au présent ou au passé, soit l'histoire, le rapport à la mémoire des événements d'une vie ordinaire. Il est donc permis de parler d'une écriture du fragmentaire empruntant des éléments de la vie ordinaire, de ce que les sujets racontent de leur vie sur le Web. La problématique de cet article est donc de comprendre la "narrativité du numérique " sur Facebook, c'est-à-dire, ce que les sujets y révèlent de leur vie, dans des textes, des parcours ou des partages - ce que nous appelons l'« écriture du fragmentaire ». Par conséquent, nous nous interrogeons sur la notion de mémoire, dans la mesure où l'écriture du fragmentaire se constitue entre la mémoire discursive et la mémoire métallique.

In the 21st century, digital relations networks outlining the poetry of the world are weaved by "clicking" web browsing and more specifically, digital writing based on images, sounds, words, 
videos blending, which constitute life as fragmentary. We are interested in these networks because the materiality of this written fabric is the everyday life, in the present or the past, that is to say history, as a relation to the memory of the ordinary life events. It is therefore possible to speak of a writing of the fragmentary, which borrows ordinary life elements, as subjects tell them on the Web. The issue of this paper is to understand the "digital narrative" on Facebook, that is to say, what subjects will reveal from their lives, in texts, courses or sharings-what we call "writing the fragmentary." Therefore, we question the notion of memory, in that the writing of the fragmentary is formed between discursive memory and metal memory.

INDEX

Mots-clés : mémoire, Facebook, narrativité, écriture du fragmentaire, numérique

Keywords : memory, narrative, fragmentary writing, digital technology

\section{AUTEUR}

\section{CRISTIANE DIAS}

Unicamp (Brésil), Labeurb 